\title{
Does Quality Control Matter? A Revisit of Surface Urban Heat Island Intensity estimated by Satellite-derived Land Surface Temperature Products
}

\author{
Jiameng Lai ${ }^{\mathrm{a}}$, Wenfeng Zhan ${ }^{\mathrm{a}, \mathrm{b} *}$, Fan Huanga \\ a Jiangsu Provincial Key Laboratory of Geographic Information Science and Technology, International Institute for Earth System \\ Science, Nanjing University, Nanjing, Jiangsu 210046, China - NJULJM@126.com; huangfan2311 @ foxmail.com \\ b Jiangsu Center for Collaborative Innovation in Geographical Information Resource Development and Application, Nanjing, 210023 , \\ China - zhanwenfeng@foxmail.com
}

ISPRS ICWG III/IVb

KEY WORDS: Surface urban heat island, Thermal remote sensing, Land surface temperature, MODIS product, Quality control

\begin{abstract}
:
Temporally regular and spatially continuous monitoring of surface urban heat island (SUHI) has been extremely difficult until the advent of spaceborne land surface temperature (LST) products. The higher errors of these LST products compared with in-situ measurements, nevertheless, have resulted in a comparatively inaccuracy and may distort the interpretation of SUHI. Although reports have shown that LST quality matters to the SUHI interpretation, a systematic investigation on how the SUHI indicators are responsive to the LST quality across cities within dissimilar bioclimates remains rare. With regard to this issue, our study chose eighty-six major cities across the mainland China and analyzed the SUHI intensity (SUHII) discrepancies (referred to as $\Delta$ SUHII) between using and not using quality control (QC) flags from Moderate Resolution Imaging Spectroradiometer data. Our major findings include: (1) the SUHII can be significantly impacted by the MODIS QC flags, and the associated seasonal $\triangle$ SUHIIs generally account for $25.5 \%$ (29.6\%) of the total intensity in the day (night). (2) The $\Delta$ SUHIIs differ season-by-season and significant discrepancies also appear among northern and southern cities, with northern ones often possessing a higher annual mean $\Delta$ SUHII. (3) The internal $\Delta$ SUHIIs within an individual city are also heterogeneous, with the variations exceeding $5.0 \mathrm{~K}(3.0 \mathrm{~K})$ in northern (southern) cities. (4) The $\Delta$ SUHII is significantly negatively related to the SUHII and cloud cover percentage mostly in transitional seasons. Our findings highlight that one needs to be very careful when using the LST-product-based SUHII to interpret the SUHI.
\end{abstract}

\section{INTRODUCTION}

More than a half of the world's population lives in urban areas, wherein natural surfaces have been altered with urban ones (WHO, 2010). One consequence of this is urban heat island (UHI), referred to as the higher urban temperature compared with rural one (Oke, 1982). The UHI appears in almost every city (Stewart and Oke, 2012) and has a lot negative impact (Taha, 1997; Sarrat et al., 2006; Grimm et al., 2008; Lafortezza et al., 2009; Tong et al., 2011; Gong et al., 2012).

The UHI has been studied using surface air temperatures obtained from weather stations for long (Morris et al., 2001; Arnfield, 2003; Shao et al., 2011). While the low density of such data hindered the UHI studies until the advent of thermal remote sensing providing periodical and spatially comprehensive coverage of land surface temperature (LST). The surface UHI (SUHI) using LST derived from thermal remote sensor has received particular attention in the recent decades (Tran et al., 2006; Yuan and Bauer, 2007; Imhoff et al., 2010; Peng et al., 2012; Zhou et al., 2014; Zhou et al., 2015; Ward et al., 2016).

To depict the satellite-derived SUHI of a city, scalar indicators were often designed. Among them, the SUHII, which is often defined as the LST difference between urban and rural areas, was considered the most widely used one (Voogt and Oke,
2003). In the SUHII estimation, many researches chose to implement a temporal composition of LST data (Gallo et al., 1993; Gallo and Owen, 1999) avoiding the impact of cloud cover. However, there still exist retrieval errors in the satellitederived LST data so that they are not of the same quality. Dealing with this, different preprocessings of the LST data were adapted in the temporal composition. Some studies disregarded that and directly use the average of the valid LSTs (Zhou et al., 2014); while others, additionally, performed a temporal aggregation of LSTs taking data quality into consideration, such as only using LSTs with good quality (Clinton,2013; Bechtel, 2015; Zhao et al, 2017), or adapting a weighted average method that the LST is weighted by the quality flags provided by the satellite products (i.e., MODIS LST QC flags (Wan, 2008)) (Zhou et al., 2013). Such different data selected methods in temporal aggregation, however, have been clarified to be able to affect the SUHII calculation even for the same city (Gawuc and Struzewska, 2016).

The SUHII variations caused by the QC flags have been preliminarily investigated, issues however persist: First, over a large scale, there remain uncertainties on the spatio-temporal variations of the SUHII due to the QC flags over cities under dissimilar bioclimates as well as the internal SUHII variations within various cities. Second, it remains unclear whether or not there exist possible controls that may affect the QC-induced SUHII variations. Without clarifications on the controls or

* Corresponding author 
factors, the decision to determine a proper algorithm of taking QC flags into consideration is hard to make.

Given these two issues, this study tries to investigate the spatiotemporal QC-induced variations of the SUHII for all the major cities in Mainland China, through which both the diurnal and seasonal variations will be examined. We will also analyze the possible factors related to these SUHII variations. Via the clarifications of the QC-induced SUHII variations at a relatively large scale, we believe this study will continue to highlight the importance and therefore help the definition of a SUHI indicator with more preciseness yet less casualness for the quantitative representation of thermal remote sensing of urban climate.

\section{STUDY AREA}

As the most populated country in the world, China has experienced a dramatic growth of population and urbanization in the past four decades, and it is projected to further continue (Seto et al., 2011; United Nations, 2014). The mainland China covers a great variety of climate zones from the tropical, to the cold climates, with the associated air temperature and precipitation generally decreasing from the south to the north (Wu et al., 2005). Cities within diverse climates give rise to a significant regional dependence for the SUHI features (e.g., intensity) (Wang et al., 1990), making the mainland China an ideal place to investigate the statistical features of SUHI at the regional/continental scale.

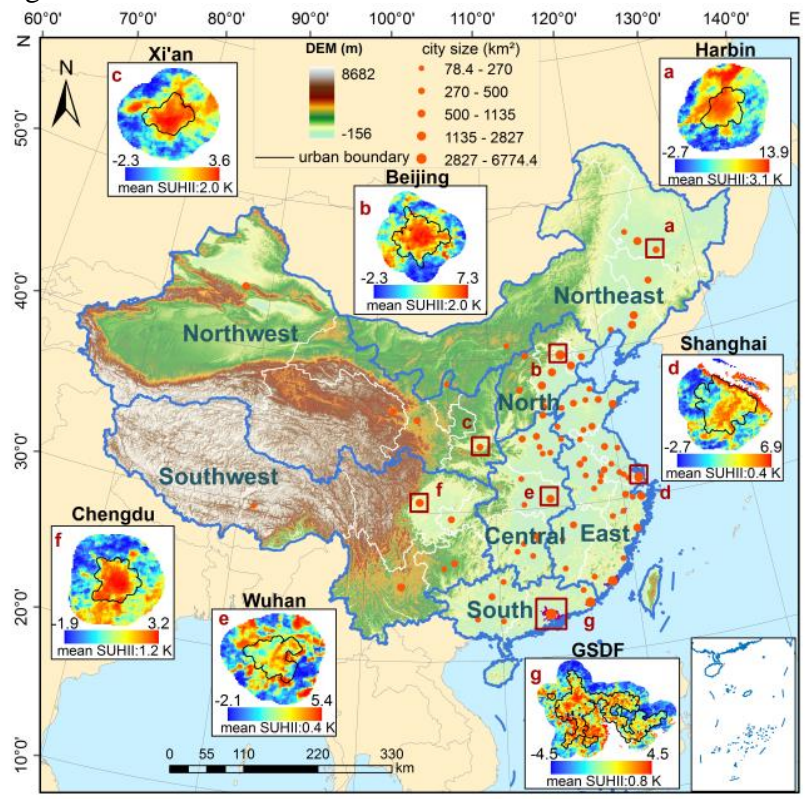

Figure 1. Geolocation of the chosen 86 cities over mainland China. Cities are given using red dots with different sizes to represent the associated city sizes. The seven selected megacities are labelled from (a) to (g). The spatial patterns of the annual mean nighttime SUHII for an entire city as well as for each pixel over these seven megacities are illustrated in the corresponding blocks.

Significant UHIs have been observed for most cities across mainland China (Li et al., 2004; Zhou and Hansen, 2004). This study selected 86 'big' cities by a combination of factors including the urban size, urban population, and the administrative significance (to make sure that a least one city is chosen for a province) (Huang et al., 2017) (see Fig. 1). Due to the terrain and climate, a great portion of these chosen cities are located in the northeastern, northern, eastern, central, and southern regions, whereas merely a small portion of cities are in the northwestern and southwestern regions. For each city, we extracted their urban areas using the MODIS land cover product, and the rural background were defined as the $15-\mathrm{km}$ buffer that surrounds the urban core, over which areas that are labelled as water bodies or featured with an elevation $\pm 50 \mathrm{~m}$ or more off the mean elevation of the urban areas were left out to eliminate the effect of water and topography (Huang et al., 2017). To better investigate the internal variations of the QC-induced SUHII within a city, we selected seven capital megacities within the seven geographical regions across mainland China. These megacities are characterized by their large urban sizes and population (with the least and largest around 8.0 and 24.2 million, respectively), economic volumes, and representativeness of the linked geographical regions. They are Harbin in northeastern China, Beijing in northern China, Xi'an in northwestern China, Shanghai in eastern China, Wuhan in central China, and Chengdu for southwestern China, the Guangzhou-Shenzhen-Dongguan-Foshan (GSDF) in southern China. Note that the GSDF denotes a metropolitan agglomeration with no clear urban boundaries shown inbetween. For these seven megacities, we also provided the spatial patterns of the annual mean nighttime SUHII in 2012 (see Fig. 1a to g).

\section{DATA AND METHOD}

\subsection{Data}

This study employed the land surface temperatures (LSTs) products retrieved from MODIS onboard Terra and Aqua satellites. The MODIS LSTs are gained at around 10:30 (Terra day), 13:30 (Aqua day), 22:30 (Terra night), and 1:30 (Aqua night) local time per day. The used LST products are the daily L3 products MOD11A1 and MYD11A1 (version 5) at the resolution of $1 \mathrm{~km}$ in 2012 .

The MODIS LSTs were retrieved using the generalized splitwindow LST algorithm (Wan and Dozier, 1996) and the associated retrieval errors have been illustrated mostly lower than $1.0 \mathrm{~K}$ (Wan, 2008). Nevertheless, the retrieval error may be higher due to a number of reasons, especially for urban areas, and such LSTs with greater uncertainties are labelled by a QC band (Wan, 1999). Each QC value is an 8-bit binary number made up of four flags representing the mandatory QA, data quality, emissivity error, and LST error. Pixels with a mandatory QA flag equal to ' 10 ' or ' 11 ' were excluded because LSTs for these pixels are invalid as a result of being covered by clouds. The LST error flag is also used, with ' 00 ', ' 01 ', ' 10 ', and ' 11 ' indicating the retrieved average LST error $\leqslant 1.0 \mathrm{~K}, \leqslant$ $2.0 \mathrm{~K}, \leqslant 3.0 \mathrm{~K}$, and $>3.0 \mathrm{~K}$, respectively.

\subsection{Method}

The temporally aggregated LSTs in a period can be estimated by the following:

$$
\hat{T}_{i}\left(t_{1}, t_{2}\right)=\frac{\int_{t_{1}}^{t_{2}} T_{i}(t) \cdot \omega_{i}(t) d t}{\int_{t_{1}}^{t_{2}} \omega_{i}(t) d t}
$$


Where $\hat{T}_{i}\left(t_{1}, t_{2}\right)=$ temporally aggregated LSTs at pixel i $t_{1}, t_{2}=$ the starting and ending times (DOY in this study) for temporal aggregation

$T_{\mathrm{i}}(t), w_{\mathrm{i}}(t)=$ original LST and its associated weight, wherein both Terra and Aqua data were included.

With the temporally aggregated LSTs, the SUHII of an entire city (i.e., $I_{c}$ ) was calculated as the difference between the mean urban and rural LSTs [Imhoff et al., 2010], while the SUHII of a certain pixel $\left(I_{i}\right)$ was estimated as the difference between the LST of this specific pixel and the mean rural LSTs, given as follows:

$$
\left\{\begin{array}{l}
I_{\mathrm{c}}\left(t_{1}, t_{2}\right)=\hat{T}_{\text {urban }}\left(t_{1}, t_{2}\right)-\hat{T}_{\text {rural }}\left(t_{1}, t_{2}\right) \\
I_{i}\left(t_{1}, t_{2}\right)=\hat{T}_{i}\left(t_{1}, t_{2}\right)-\hat{T}_{\text {rural }}\left(t_{1}, t_{2}\right)
\end{array}\right.
$$

Where $\hat{T}_{\text {urban }}, \hat{T}_{\text {rural }}=$ the weighted mean urban and rural LSTs.

To obtain temporally aggregated SUHIs, a portion of the previous studies weighted all the clear-sky LSTs at different times equally regardless of their quality (hereafter termed the equally weighted aggregation (EWA) strategy) $[\mathrm{Hu}$ and Brunsell, 2013; Quan et al., 2014]. By comparison, others achieved the temporal aggregation through weighting the clearsky LSTs by the QC flags (hereafter termed the unequally weighted aggregation (UWA) strategy) [Zhou et al., 2013; Gawuc and Struzewska, 2016]. This study estimated the weights of the UWA as the inverse-square of the retrieved LST error [Zhou et al., 2013]. Therefore, the EWA and UWA approaches can be written as follows:

$$
\left\{\begin{array}{l}
\omega_{\text {ewa }}=1 \\
\omega_{\text {uwa }}=q^{-2}
\end{array}\right.
$$

Where $\omega_{\text {ewa }}, \omega_{\text {uwa }}=$ weights given by the EWA and UWA strategies:

$$
q=\text { the retrieved LST error provided in QC flags. }
$$

The values of $q$ were designated as 1.0, 2.0, 3.0, and infinitely great, to represent the cases with the LST errors of $\leq 1.0 \mathrm{~K}, \leq 2.0$ $\mathrm{K}, \leq 3.0 \mathrm{~K}$, and $>3.0 \mathrm{~K}$, respectively. This setting suggests that the weight is equal to zero once the LST error is greater than 3.0 K.

The QC-induced SUHII variation ( $\triangle \mathrm{SUHII})$ was therefore quantified as the difference between using $\omega_{\text {ewa }}$ and $\omega_{\text {uwa }}$ as the weights for temporal aggregation, written as follows:

$$
\Delta \operatorname{SUHII}\left(t_{1}, t_{2}\right)=I_{\text {ewa }}\left(t_{1}, t_{2}\right)-I_{\text {uwa }}\left(t_{1}, t_{2}\right)
$$

Where $I_{\text {ewa }}, I_{\text {uwa }}=$ the SUHIIs without and with considering the QC flags.

\section{RESULTS}

The resulted $\Delta$ SUHIIs for each city are demonstrated season-byseason for both the daytime and nighttime. The internal spatial variations of the annual mean $\Delta$ SUHII are also further provided for the chosen seven megacities.

\subsection{Daytime}

The spatial distributions of $\triangle$ SUHII over mainland China during the four seasons are provided in Fig. 2. The result shows that the $\Delta$ SUHII values are different city-by-city and season-byseason. Generally, the mean absolute $\Delta$ SUHII for all these cities in each season is $0.44 \mathrm{~K}$, with the maximum $\Delta$ SUHII appearing in Xi'an (northwest) during the winter $(3.1 \mathrm{~K})$ and the minimum occurring in Wuxi (east) during the autumn $(-1.1 \mathrm{~K})$. Though the mean value $(0.44 \mathrm{~K})$ seems not large for the SUHII of a city, our data illustrate that 75/86 (20/86) of these cities possessed a $\Delta$ SUHII exceeding $\pm 0.5 \mathrm{~K}( \pm 1.0 \mathrm{~K})$ in a certain season during a year. Such results indicate that the difference between using and not using the MODIS QC flags can be significant and that the weighted QC-based (wqc) method is able to make a difference to SUHII that is not ignorable.

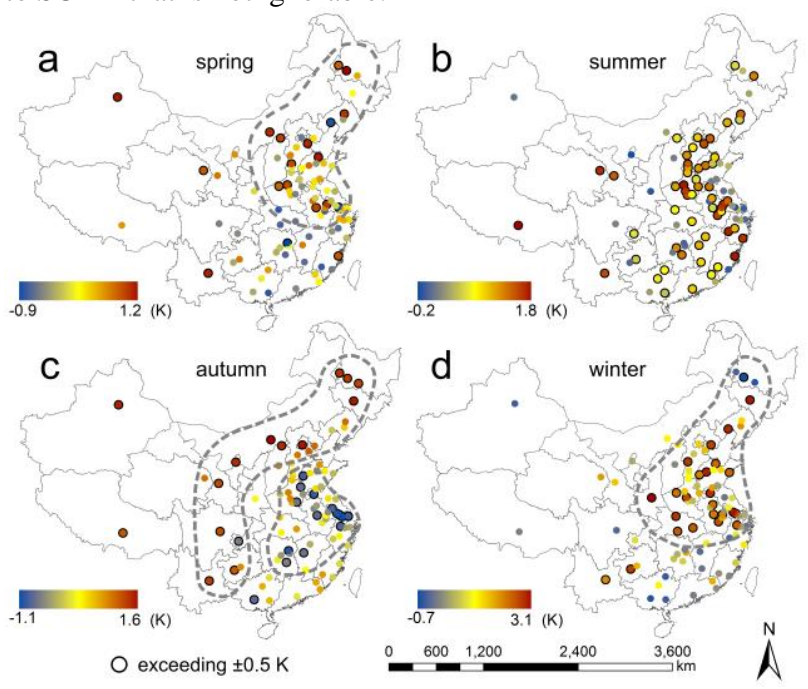

Figure 2. Spatial pattern of $\Delta$ SUHII (K) during the day for the four seasons. Regions where most cities have a significant $\Delta$ SUHII are highlighted with grey dotted curve box.

During the spring, the $\Delta$ SUHII ranges from -0.9 to $1.2 \mathrm{~K}$, and $19 / 86(2 / 86)$ of these cities possessed a $\Delta$ SUHII exceeding $\pm 0.5 \mathrm{~K}( \pm 1.0 \mathrm{~K})$ The cities with $\mathrm{SUHII}_{\text {eqc }}$ significantly higher than $\mathrm{SUHII}_{\mathrm{wqc}}(\Delta \mathrm{SUHII}>+0.5 \mathrm{~K})$ are mostly located in the northern and northeastern regions, with a few located in the other regions (see Fig. 2a). By comparison, only three cities are characterized by a $\Delta \mathrm{SUHII}<-0.5 \mathrm{~K}$. During the summer, the maximum and minimum $\Delta$ SUHII are $1.8 \mathrm{~K}$ (Lhasa, southwest) and $-0.2 \mathrm{~K}$ (Yinchuan, northwest), respectively, and $56 / 86(7 / 86)$ of these cities had a $\Delta$ SUHII exceeding $\pm 0.5 \mathrm{~K}$ $( \pm 1.0 \mathrm{~K})$, indicating that significant differences occur in more than a half of the cities. It is also interesting that the majority of cities had a $\Delta$ SUHII higher than zero (only three cities had a $\Delta$ SUHII lower than zero) (Fig. 2b). In other words, for most of the cities, the SUHII ${ }_{\text {eqc }}$ is higher than the SUHII wqc. Analyses by checking the data show that, during the summer, higher LSTs were often labeled with lower retrieval errors, especially in the rural areas since the lowest flagged errors, i.e., $\mathrm{QC}=1$, tend to appear more in the rural pixels, and as a result leading to a slightly increased mean urban LST and significantly increased mean rural LST when weighted by the QC flag, lowering the associated SUHII (i.e., the SUHII ${ }_{\text {uwa }}$ decreases), and consequently contributing to the significantly positive $\Delta$ SUHII.

During the autumn, the maximum and minimum $\triangle \mathrm{SUHII}$ are $1.6 \mathrm{~K}$ (Hohhot, northeast) and $-1.1 \mathrm{~K}$ (Wuxi, east), respectively, 
and $30 / 86(9 / 86)$ of these cities had a $\Delta$ SUHII exceeding $\pm 0.5 \mathrm{~K}$ $( \pm 1.0 \mathrm{~K})$. The cities with $\Delta$ SUHII $>+0.5 \mathrm{~K}$ are mostly located in the northeastern, northern, and western regions, while those with $\Delta \mathrm{SUHII}<-0.5 \mathrm{~K}$ are mainly situated in eastern and southern regions (see Fig. 2c). During the winter, the $\Delta$ SUHII ranges from -0.7 to $3.1 \mathrm{~K}$, and $25 / 86(5 / 86)$ of these cities had a $\triangle$ SUHII exceeding $\pm 0.5 \mathrm{~K}( \pm 1.0 \mathrm{~K})$. The cities with $\Delta$ SUHII $>+0.5 \mathrm{~K}$ are mostly distributed in the eastern, northern, and northeastern regions, with only two exceptions that are located in the western region. By comparison, there is only a single city possessing a $\Delta \mathrm{SUHII}<-0.5 \mathrm{~K}$ (i.e., Daqing in the northeastern region).

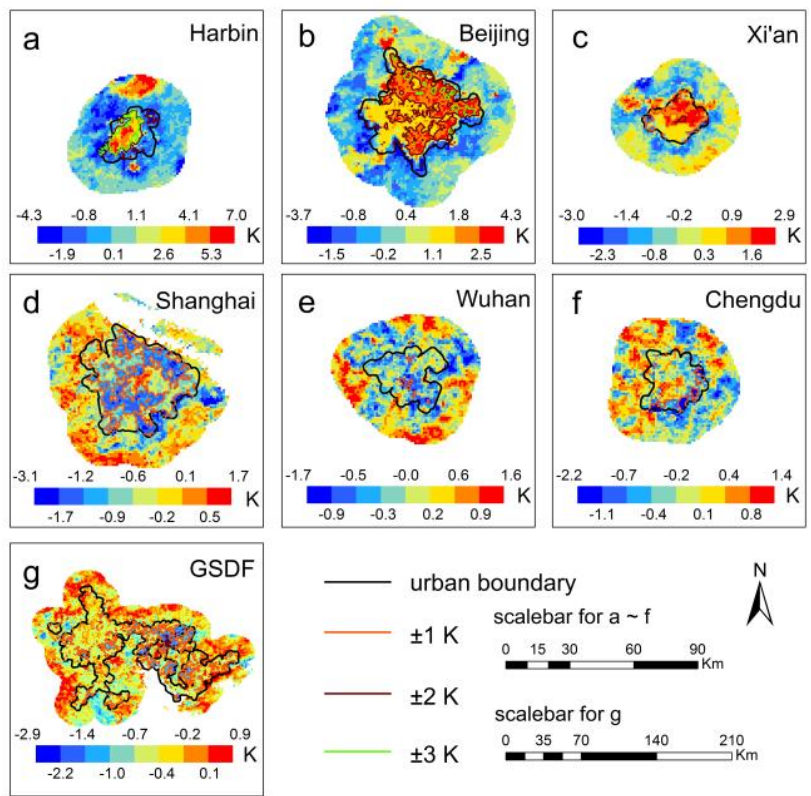

Figure 3. Spatial distributions of the annual mean daytime $\Delta$ SUHII within the chosen seven cities. Urban areas (within the black lines) and rural areas within a $15-\mathrm{km}$ buffer zone are shown for each city.

Considering that (1) one may argue that the $\Delta$ SUHII remains not large (around 0.5 to $1.0 \mathrm{~K}$ ) for the quantification of the SUHI, and (2) the use of a single SUHII to represent the thermal status of an entire city is insufficient, we therefore further provide the internal spatial patterns of the annual mean daytime $\Delta$ SUHII within the chosen seven cities (see Fig. 3). Calculations show that the annual mean daytime $\Delta$ SUHIIs are $0.5,1.3,0.5,-0.6,-0.1,-0.3$, and $-0.2 \mathrm{~K}$ for Harbin, Beijing, Xi'an, Shanghai, Wuhan, Chengdu, and GSDF, respectively. Nevertheless, for the internal patterns of the $\Delta$ SUHII within a city, the pixel-by-pixel $\Delta$ SUHIIs are not uniformly distributed high spatial heterogeneity can be observed. They vary from -4.3 to $7.0 \mathrm{~K}$ for Harbin, from -3.7 to $4.3 \mathrm{~K}$ for Beijing, from -3.0 to $2.9 \mathrm{~K}$ for $\mathrm{Xi}$ 'an, from -3.1 to $1.7 \mathrm{~K}$ for Shanghai, from -1.7 to $1.6 \mathrm{~K}$ for Wuhan, from -2.0 to $1.4 \mathrm{~K}$ for Chengdu, and from -2.9 to $0.9 \mathrm{~K}$ for GSDF. Such results confirm that the pixel-bypixel variations of the differences between the SUHIIeqc and SUHII $_{\text {wqc }}$ are much higher than the city-averaged difference.

The results given by Figs. 3 also indicate that the internal $\Delta$ SUHIIs for the northern cities generally differ from those for the southern in the following two regards:

First, for the three northern cities, the urban $\Delta$ SUHIIs are generally higher than the rural ones, while for the four southern cities, the opposite phenomenon appears - with the urban $\Delta$ SUHIIs largely lower than the rural (see Figs. 3). The significantly higher annual mean $\Delta$ SUHIIs over northern cities can be evidenced by the spatial patterns given in Fig. 3. Further analyses by checking the data confirm that a high $\Delta$ SUHII is likely caused by the aggregation of low-quality LST pixels yet with relatively high values (i.e., around summer) weakening the intensity calculated using the QC flags (SUHII $\left.{ }_{w q c}\right)$. By comparison, a very low (negative) $\Delta$ SUHII can be caused by high-quality LSTs mainly aggregate in summer and/or lowquality LSTs are collectively located around winter.

Second, the spatial ranges of the relatively higher $\Delta$ SUHIIs over the northern cities commonly correspond to their urban boundaries (Fig. 3b), but this close correspondence does not exist for the southern cites (Fig. 3e). In addition, for the northern cities, the $\triangle$ SUHIIs over the western areas within Harbin, the northern, eastern, and southern areas within Beijing, and the northern areas within $\mathrm{Xi}$ 'an, are still higher than those over the other areas. Comparatively, for the southern cities, there remain small areas characterized by local high $\Delta$ SUHIIs, e.g., the very center of Shanghai as well as the very center and southwestern areas of Chengdu.

\subsection{Nighttime}

The spatial distribution of the seasonal $\Delta$ SUHII during the night are shown in Figs. 4. In general, the $\Delta$ SUHII values can also be significant in the night. The mean absolute $\Delta$ SUHII during the night is $0.38 \mathrm{~K}$, and the number of cities possessing a $\Delta$ SUHII exceeding $\pm 0.5 \mathrm{~K}( \pm 1.0 \mathrm{~K})$ in a certain season is $55 / 86(20 / 86)$, which are lower than those values during the day but they also imply evident impacts from MODIS QC flags on SUHII.

During the night, significant differences between the southern and northern regions can be observed in the two transitional seasons (i.e., spring and autumn) (Fig. 4); such a spatial contrast, however, does not occur in the summer and winter, within which there are only minimum differences (the $\triangle$ SUHII is close to zero). The results in Fig. 4 further illustrate that, differing from the day, during the night the MODIS QC produces a larger impact in the two transitional seasons, within which the maximum ( $2.4 \mathrm{~K}$ for the spring) and minimum $(-0.9 \mathrm{~K}$ for the autumn) $\triangle$ SUHII values appear, and within which there are higher absolute mean values that reach $0.6 \mathrm{~K}$.

In the spring, the highest and lowest $\Delta$ SUHII values are $2.4 \mathrm{~K}$ (Beijing, north) and $-0.5 \mathrm{~K}$ (Chongqing, southwest, also the only value lower than $-0.5 \mathrm{~K})$ respectively, and $41 / 86(18 / 86)$ cities have an absolute $\Delta$ SUHII value exceeding $\pm 0.5 \mathrm{~K}( \pm 1.0$ $\mathrm{K})$. The cities with a $\Delta$ SUHII value higher than $+0.5 \mathrm{~K}$ can be found in all the seven geographic regions, with most of them located in the northern and northeastern regions (Fig. 4a). In the summer, the maximum and minimum $\triangle$ SUHII values are $0.7 \mathrm{~K}$ (Xining, northwest) and $-0.7 \mathrm{~K}$ (Taiyuan, north), indicating that there is no city that has a $\Delta$ SUHII value exceeding $\pm 1.0 \mathrm{~K}$. Only four cities possess a $\Delta$ SUHII value exceeding $\pm 0.5 \mathrm{~K}$ (three of them have a positive $\Delta$ SUHII while only one has a negative one) (Fig. 4b). In the autumn, the corresponding maximum and minimum are $1.9 \mathrm{~K}$ (Beijing, north) and $-0.9 \mathrm{~K}$ (Nanjing, east), respectively, and $41 / 86(12 / 86)$ cities have a $\Delta$ SUHII value surpassing $\pm 0.5 \mathrm{~K}( \pm 1.0 \mathrm{~K})$. Cities with the $\Delta$ SUHII value higher than $+0.5 \mathrm{~K}$ are mostly situated in the northern, northeastern and northwestern regions, while only six cities show a value lower than $-0.5 \mathrm{~K}$, with five of situated in the eastern region and one situated in the southern region (Fig. 4c). In the winter, the maximum and minimum are $0.5 \mathrm{~K}$ (Heze, east) and $-0.5 \mathrm{~K}$ (Fuzhou, east), respectively, and these two cities are also the only two with the $\Delta$ SUHII exceeding $\pm 0.5 \mathrm{~K}$. In other 
words, the MODIS QC flags present a less significant impact on the calculation of SUHII in the winter night (Fig. 4d).

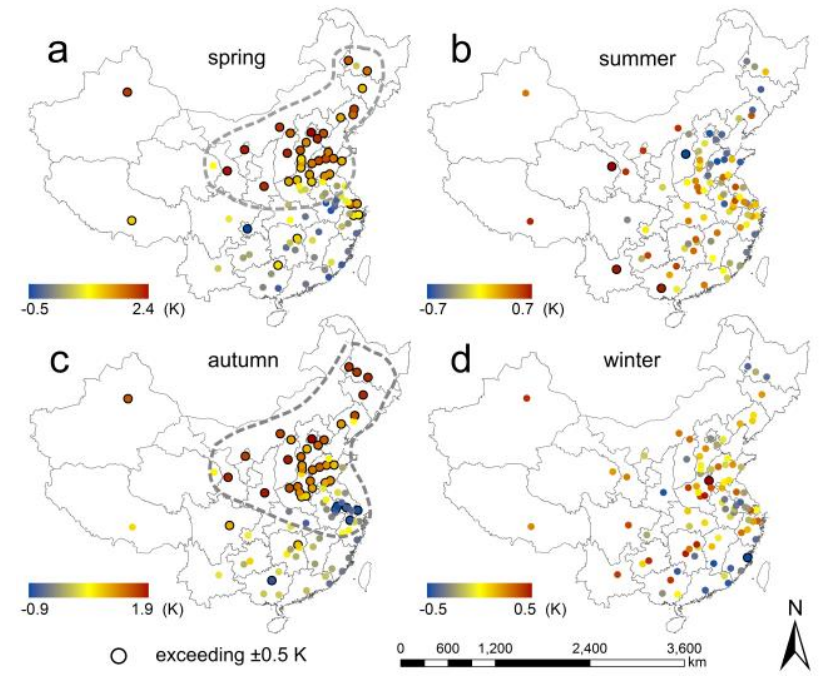

Figure 4. Spatial pattern of $\Delta$ SUHII (K) during the night for the four seasons. Regions where most cities have a significant $\Delta$ SUHII are highlighted with grey dotted curve box.

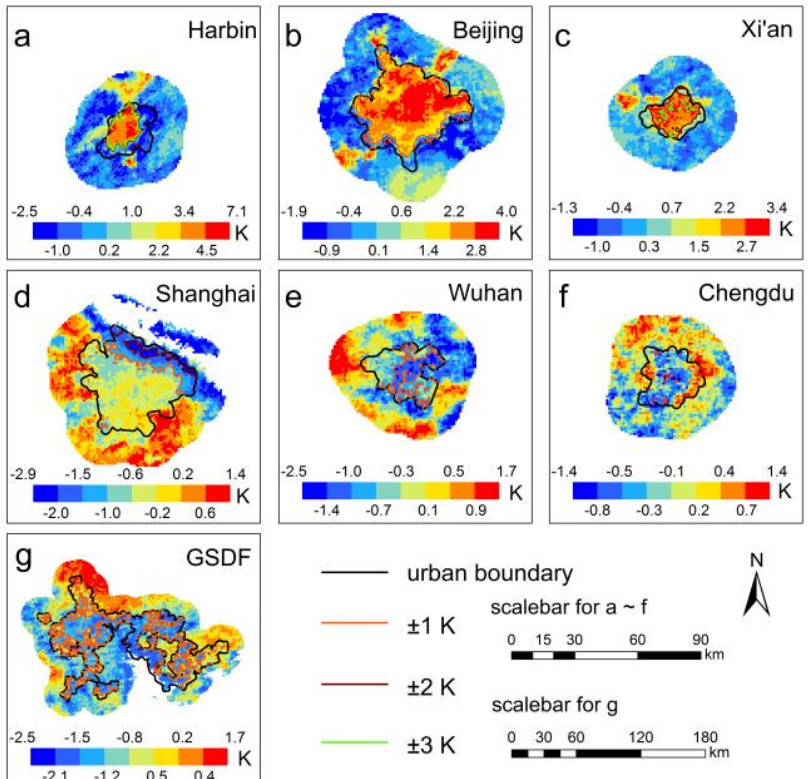

Figure 5. Spatial distributions of the annual mean $\Delta$ SUHII within the chosen seven cities during the night. Urban areas (within the black lines) and rural areas within a $15-\mathrm{km}$ buffer zone are shown for each city.

The internal spatial patterns of the annual mean nighttime $\Delta$ SUHII within the chosen seven cities are provided in Fig. 5. The annual mean $\Delta$ SUHIIs for Harbin, Beijing, Xi'an, Shanghai, Wuhan, Chengdu, and GSDF are 1.7, 1.9, 2.1, -0.6, -0.7, 0.1, and $-0.3 \mathrm{~K}$, respectively. High spatial heterogeneity was also found in the night on par with that of daytime, with the range varying from -2.5 to $7.1 \mathrm{~K}$ in Harbin, from -1.9 to $4.0 \mathrm{~K}$ in Beijing, from -1.3 to $3.4 \mathrm{~K}$ in $\mathrm{Xi}$ 'an, from -2.9 to $1.4 \mathrm{~K}$ in Shanghai, from -2.5 to $1.7 \mathrm{~K}$ in Wuhan, from -1.4 to $1.4 \mathrm{~K}$ in Chengdu, and from -2.5 to $1.7 \mathrm{~K}$ in GSDF.

During the night, the $\Delta$ SUHII contrast between the three northern and four southern cities is similar to that of the daytime: First, the urban nighttime $\Delta$ SUHIIs are also higher over the three northern cities, while the opposite phenomenon appears in the southern cities (Fig. 5). Second, the consistency between the spatial ranges of high $\Delta$ SUHIIs and urban boundaries is also illustrated mainly in northern cities (Fig. 5), while such consistency is in absence for the southern cities.

The characteristic similarities between the daytime and nighttime indicate that the spatial patterns of the annual mean $\Delta$ SUHII within an individual city are largely insensitive to the hour of day. We however need to emphasize that dissimilarities do exist. For example, the $\Delta$ SUHIIs within urban Shanghai are much more homogeneous during the night than the day (see Figs. 3d and 5d).

\section{DISCUSSION}

\subsection{Factors related to $\Delta$ SUHII}

By definition, the spatio-temporal $\Delta$ SUHII variations as shown in Section 4 are related to two groups of factors. They include those on the QC flag and on the SUHI features. The following displays the relationships between the $\triangle \mathrm{SUHII}$ and one factor related to the QC flag (i.e., the cloud cover percentage, $\mathrm{CCP}$ ) as well as one factor related to the SUHI features (i.e., the SUHII).

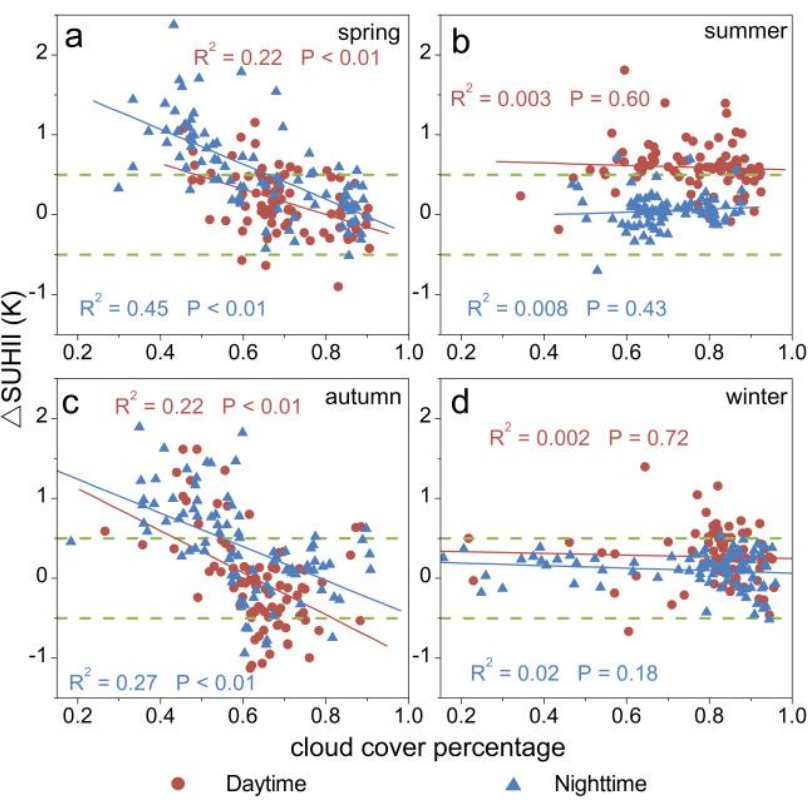

Figure 6. Relationships between the $\triangle$ SUHII and cloud percentage during the four seasons for both daytime and nighttime across the chosen 86 cities in Mainland China.

The relationships between the $\triangle$ SUHII and CCP over all the chosen cities are given in Fig. 6. These scatters indicate that the $\triangle$ SUHII is negatively and linearly related to the CCP in the two transitional seasons, all with the p-value at the 0.01-level. Such a significant association however disappears for the two nontransitional seasons (i.e., summer and winter). Noteworthy to discern is that, for the two transitional seasons, cites with a relatively lower CCP (with a value around 0.4 or lower) are likely to demonstrate a higher and positive $\Delta$ SUHII. By comparison, very few cites in spring and only a small part of cities in autumn, exhibit a relatively low $\triangle$ SUHII that exceeding $-0.5 \mathrm{~K}$ when the CCP is high (with a value around 0.6 to 0.9 ). This may be attributed to that the $\Delta$ SUHII is more related to the temporal distribution of the QC flags rather than the absolute value of the CCP. 
The relationships between the $\Delta$ SUHII and SUHII (calculated in wqc method) are provided in Fig. 7. These results, once more, show that the $\triangle$ SUHII is negatively correlated to the SUHII in the two transitional seasons (i.e., spring and autumn) for both daytime and nighttime, as well as in the winter daytime, with the associated p-values at the 0.01- or 0.05-level. By comparison, there is no statistical correlation in the summer and nighttime winter. Such a contrast of statistical significance, largely between the transitional and non-transitional seasons, is as well similar to that provided in Fig. 6; it is also well consistent with the spatio-temporal patterns of the $\Delta$ SUHII given in Figs. 2 and 4 . In other words, the $\Delta$ SUHII is strongly related to the SUHII when substantial $\Delta$ SUHII differences between the southern and northern cities are observed. The contrast of significance among different seasons is partly because LSTs in the transitional seasons typically keep a continually decreasing (in autumn) or an increasing (in spring) trend, which makes the QC flags generate a greater effect on the $\Delta$ SUHII and therefore leads to a higher $\Delta$ SUHII variance. Besides, there were evidences that demonstrated that the SUHII itself also demonstrated a latitudinal contrast for the major cities in mainland China (Zhou et al., 2014), which likely contributes to the significant relationships between the $\Delta$ SUHII and SUHII.

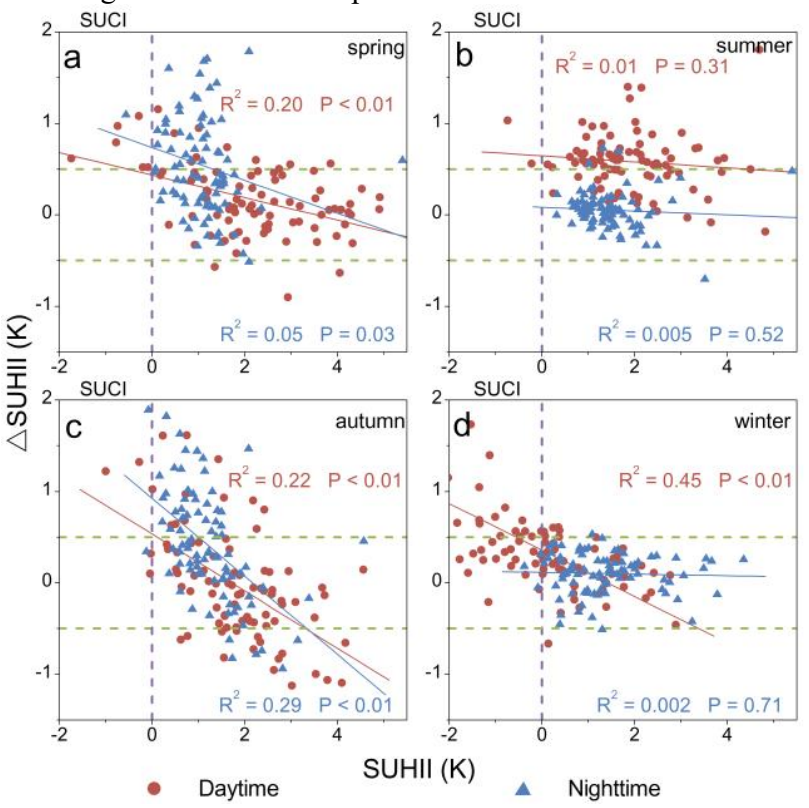

Figure 7. Relationships between the $\Delta$ SUHII and SUHII during the four seasons for both daytime and nighttime across the chosen 86 cities in Mainland China.

The correlations in Fig. 7 further indicate that, in the two transitional seasons, relative high $\Delta$ SUHIIs (higher than $0.5 \mathrm{~K}$ ) mainly appear in the cities with a small or negative SUHII, with the latter indicating a surface urban cool island (SUCI) occurs (Clinton and Peng, 2013; Lazzarini et al., 2013; Haashemi et al., 2016). During these transitional periods, significant but negative $\Delta$ SUHIIs (lower than $-0.5 \mathrm{~K}$ ) can also appear in cities with a high SUHII. In the daytime winter, cities characterized by a SUCI possess a $\triangle$ SUHII greater than $0.5 \mathrm{~K}$, but such a phenomenon disappears in the nighttime winter.

Though the $\triangle$ SUHII may be related to the CCP/SUHII, their accompanying $\mathrm{R}^{2}$ are not high, with the highest values only equal to 0.45 in the winter daytime. This is because the QC flags were processed more complicatedly than considering the $\mathrm{CCP}$; they as well are determined by a combination of the radiometric and geometric accuracy of MODIS products, confidence in land cover classification and its associated emissivity uncertainty, topographic effect and mixture-pixel effect, along with others (Wan, 1999). In addition to these controls, the $\triangle \mathrm{SUHII}$ is supposed to be further related to temporal distribution of the QC flags as well as the SUHIIrelated controls, including the background climate, rural background, and surface characteristics of a city (such as the fractions between urban vegetation and impervious surfaces) (Wang et al., 2007; Yuan and Bauer, 2007). Despite a quantitative analysis between the $\triangle$ SUHII and these abovementioned controls is difficult to conduct, we nevertheless may infer that these factors interplay and they together possibly explain the spatio-temporal variations of the $\Delta$ SUHII.

\subsection{Issues related to the true SUHII}

This study primarily estimated several kinds of SUHII and provided their differences for the major cities over mainland China. According to the analyses in Sections 4 and 5.1, the general differences $(\triangle \mathrm{SUHII})$ are able to reach $25.5 \%$ and $29.6 \%$ of the total intensity in the daytime and nighttime, respectively, one thus needs to be very cautious when using the estimated SUHII, whether the SUHII eqc or SUHII wqc $_{\text {, of a }}$ certain city. The magnitude of these variations is on the par with the possible SUHII variation produced owing to surface thermal anisotropy ( $\mathrm{Hu}$ et al., 2016). We further acknowledge that different SUHI indicators including the SUHII, may be inconsistent with each other on the representation of the UHI feature even for the same city (Schwarz et al., 2011). We as well understand that the SUHII may further suffer from its casual definition, such as the subjectivity of choosing the associated rural background and the disregard of the differences among different urban local climate zones (Stewart and Oke, 1982). Nevertheless, to facilitate the communications within research communities, the SUHII simply defined from the dichotomy, whether from the traditional view or latest perspective, remains straightforward yet indispensable to depict the global feature of the SUHI of a certain city. (Quan et al., 2014)

In view of the still-existing necessity of using the SUHII, its variations due to three factors including (1) surface thermal anisotropy, (2) missing data caused by clouds, as well as (3) QC flags as reported in this study, require to be corrected. The SUHII alterations owing to thermal anisotropy require a semiempirical model that is capable of correcting the off-nadir thermal observations into the nadir direction, as that has done for directional reflectance in the optical spectra, which currently seems possible for geostationary thermal observations (Vinnikov et al., 2012) but remains in its infancy for thermal observations from polar-orbiting satellites (Li et al., 2013). Similarly, it will be challenging to adjust the SUHII variations and their temporally aggregated values in a period as a result of data missing and QC flags. In theory, the accurate correction of variations due to the latter two factors both require the reconstruction of LST dynamics over urban and rural areas by the nonlinear annual temperature cycle (ATC) and/or diurnal temperature cycle (DTC) models, as those that have been preliminarily conducted by Huang et al. (2016). Such a correction also demands the design of an algorithm that could better set the weights of the LST in temporal aggregations according to the nonlinear LST dynamics and the temporal distribution of the different QC flags, in addition to the predetermined absolute errors of retrieved LSTs. 


\section{CONCLUSIONS}

The understanding of surface urban heat island (SUHI) has greatly benefited from the satellite-derived land surface temperature (LST) products, but the drawbacks of such products also cause detriment to and even distort the interpretation of SUHI. Specifically, as the most basic indicator to describe the SUHI, the SUHI intensity (SUHI), once estimated using satellite-derived LST products, is not consistent between considering or not the quality control (QC) of these products. To investigate the variations of the SUHII along with the LST quality across cities within different climates, this study chose eighty-six megacities across the mainland China and analyzed the associated spatio-temporal patterns of the QCinduced SUHII variations (termed $\Delta$ SUHII).

Our results generally show that the QC flags have a large impact on the SUHII, which can account for $25.5 \%$ (29.6\%) of the total intensity in the day (night). More elaborate conclusions are summarized as below: First, the $\Delta$ SUHII differs city-by-city and the pixel-based $\Delta$ SUHII also has a high variation within a city. The amplitude of the daytime (nighttime) $\Delta$ SUHII variations across cities during a certain season can reach $3.8 \mathrm{~K}(2.9 \mathrm{~K})$; and there is a significant discrepancy among northern and southern cities, with the northern ones possessing generally higher $\Delta$ SUHIIs, mostly during the two transitional seasons. For the seven chosen cities, the amplitude of variation for the annual mean pixel-based $\Delta$ SUHIIs exceeds $5.0 \mathrm{~K}(3.0 \mathrm{~K})$ in northern (southern) cities. Second, the $\Delta$ SUHII differs seasonby-season and shows a strong day/night contrast. In the daytime, the mean $\Delta$ SUHIIs for the two transitional seasons (spring and autumn) are relatively closer to zero compared with those for the two extreme seasons (summer and winter). However, the opposite phenomenon occurs in the nighttime, during which the mean $\Delta$ SUHIIs for the two extreme seasons are closer to zero. Besides, the $\Delta$ SUHII across cities shows a greater variation in the two transitional seasons than in the two extreme seasons, both in the daytime and nighttime. Finally, the $\triangle$ SUHII is negatively related to the cloud cover percentage (CCP) and SUHII, which however only appears in the transitional seasons.

Inadequacies of this study also remain. First, this study was conducted at the temporal scale of a season (the annual mean values were provided but they were only for the inner-city pixels), while the $\Delta$ SUHII variations at other temporal aggregation scales such as the month are unconsidered. Second, our analyses were based on the most frequently used SUHI indicator (i.e., SUHI intensity), while how the other SUHI indicators (e.g., the hot island area) will respond to the QC flags, remain unexplored. Though with these inadequacies, our study vividly highlights that, to interpret the SUHI, one need to be very careful when using the SUHII calculated based on satellitederived LSTs. Our investigation further underlines the necessity of defining a SUHI indicator independent of the temporally missing data and quality control flags.

\section{ACKNOWLEDGMENTS}

The used MODIS data were downloaded at NASA's Earth Observing System and Data and Information System (EOSDIS) (https://earthdata.nasa.gov/). This work was supported in part by the National Natural Science Foundation of China under Grants 41301360 and 41671420 , and by the Key Research and Development Programs for Global Change and Adaptation under 2016YFA0600201. We also thank the financial support from the DengFeng Program-B of Nanjing University.

\section{REFERENCES}

Arnfield, A. J., 2003. Two decades of urban climate research: a review of turbulence, exchanges of energy and water, and the urban heat island. International Journal of Climatology, 23(1), pp. 1-26.

Bechtel, B., 2015. A new global climatology of annual land surface temperature. Remote Sensing, 7(3), pp. 2850-2870.

Clinton, N., and G. Peng, 2013. MODIS detected surface urban heat islands and sinks: Global locations and controls. Remote Sensing of Environment, 134(5), pp. 294-304.

Gallo, K. P., A. L. McNab, T. R. Karl, J. F. Brown, J. J. Hood, and J. D. Tarpley, 1993. The use of NOAA AVHRR data for assessment of the urban heat island effect. Journal of Applied Meteorology, 32(5), pp. 899-908.

Gallo, K. P., and T. W. Owen, 1999. Satellite-based adjustments for the urban heat island temperature bias. Journal of Applied Meteorology, 38(6), pp. 806-813.

Gawuc, L., and J. Struzewska, 2016. Impact of MODIS quality control on temporally aggregated urban surface temperature and long-term surface urban heat island intensity. Remote Sensing, 8(5), pp. 374.

Gong, P., S. Liang, E. J. Carlton, Q. Jiang, J. Wu, L. Wang, and J. V. Remais, 2012. Urbanisation and health in China. The Lancet, 379(9818), pp. 843-852.

Grimm, N. B., S. H. Faeth, N. E. Golubiewski, C. L. Redman, J. Wu, X. Bai, and J. M. Briggs, 2008. Global change and the ecology of cities, Science, 319(5864), pp. 756-760.

Haashemi, S., Q. Weng, A. Darvishi, and S. Alavipanah, 2016. Seasonal variations of the surface urban heat island in a semiarid city. Remote Sensing, 8(4).

Hu, L., and N. A. Brunsell, 2013. The impact of temporal aggregation of land surface temperature data for surface urban heat island (SUHI) monitoring. Remote Sensing of Environment, 134, pp. 162-174.

Hu, L., A. Monaghan, J. A. Voogt, and M. Barlage, 2016. A first satellite-based observational assessment of urban thermal anisotropy. Remote Sensing of Environment, 181, pp. 111-121.

Huang, F., W. Zhan, J. Voogt, L. Hu, Z. Wang, J. Quan, W. Ju, and Z. Guo, 2016. Temporal upscaling of surface urban heat island by incorporating an annual temperature cycle model: A tale of two cities. Remote Sensing of Environment, 186, pp. 112.

Huang, F., W. Zhan, K. Wang, J. Chen, and W. Ju, 2017. A Satellite perspective into urbanization-induced variation in diurnal skin-surface temperature range for major cities across mainland china. Geophysical Research Letters, in submission.

Imhoff, M. L., P. Zhang, R. E. Wolfe, and L. Bounoua, 2010. Remote sensing of the urban heat island effect across biomes in the continental USA. Remote Sensing of Environment, 114(3), pp. 504-513.

Lafortezza, R., G. Carrus, G. Sanesi, and C. Davies, 2009. Benefits and well-being perceived by people visiting green spaces in periods of heat stress. Urban Forestry \& Urban Greening, 8(2), pp. 97-108.

Lazzarini, M., P. R. Marpu, and H. Ghedira, 2013. Temperature-land cover interactions: The inversion of urban heat island phenomenon in desert city areas. Remote Sensing of Environment. 130(4), pp. 136-152.

Li, Q., H. Zhang, X. Liu, and J. Huang, 2004. Urban heat island effect on annual mean temperature during the last 50 years in 
China. Theoretical and Applied Climatology, 79(3), pp. 165174.

Li, Z., B. Tang, H. Wu, H. Ren, G. Yan, Z. Wan, I. F. Trigo, and J. A. Sobrino, 2013. Satellite-derived land surface temperature: Current status and perspectives. Remote Sensing of Environment, 131(8), pp. 14-37.

Morris, C. J. G., I. Simmonds, and N. Plummer, 2001. Quantification of the influences of wind and cloud on the nocturnal urban heat island of a large city. Journal of Applied Meteorology, 40(2), pp. 169-182.

Oke, T. R., 1982. The energetic basis of the urban heat island. Quarterly Journal of the Royal Meteorological Society, 108(455), pp. 1-24.

Peng, S., S. Piao, P. Ciais, P. Friedlingstein, C. Ottle, F. M. Breon, H. Nan, L. Zhou, and R. B. Myneni, 2012. Surface urban heat island across 419 global big cities. Environmental science \& technology, 46(2), pp. 696-703.

Quan, J., Y. Chen, W. Zhan, J. Wang, J. Voogt, and M. Wang, 2014. Multi-temporal trajectory of the urban heat island centroid in Beijing, China based on a Gaussian volume model. Remote Sensing of Environment, 149(7), pp. 33-46.

Sarrat, C., A. Lemonsu, V. Masson, and D. Guedalia, 2006. Impact of urban heat island on regional atmospheric pollution. Atmospheric Environment, 40(10), pp. 1743-1758.

Schwarz, N., S. Lautenbach, and R. Seppelt, 2011. Exploring indicators for quantifying surface urban heat islands of European cities with MODIS land surface temperatures. Remote Sensing of Environment, 115(12), pp. 3175-3186.

Seto, K. C., M. Fragkias, B. Güneralp, and M. K. Reilly, 2011. A meta-analysis of global urban land expansion. PLOS One, 6(8), p. e23777.

Shao, Q., C. Sun, J. Liu, J. He, W. Kuang, and F. Tao, 2011. Impact of urban expansion on meteorological observation data and overestimation to regional air temperature in China. Journal of Geographical Sciences, 21(6), pp. 994-1006.

Stewart, I. D., and T. R. Oke, 2012. Local climate zones for urban temperature studies. Bulletin of the American Meteorological Society, 93(12), pp. 1879-1900.

Taha, H., 1997. Urban climates and heat islands: albedo, evapotranspiration, and anthropogenic heat. Energy and Buildings, 25(2), pp. 99-103.

Tong, N. Y. O., D. Y. C. Leung, and C.-H. Liu, 2011. A review on ozone evolution and its relationship with boundary layer characteristics in urban environments. Water, Air, \& Soil Pollution, 214(1), pp. 13-36.

Tran, H., D. Uchihama, S. Ochi, and Y. Yasuoka, 2006. Assessment with satellite data of the urban heat island effects in Asian mega cities. International Journal of Applied Earth Observation and Geoinformation, 8(1), pp. 34-48.

United Nations, 2015. World urbanization prospects: the 2014 revision, Rep.

Vinnikov, K. Y., Y. Yu, M. D. Goldberg, T. Dan, P. Romanov, I. Laszlo, and M. Chen, 2012. Angular anisotropy of satellite observations of land surface temperature. Geophysical Research Letters, 39(23), p. L23802.

Voogt, J. A., and T. R. Oke, 2003. Thermal remote sensing of urban climates. Remote Sensing of Environment, 86(3), pp. 370384.

Wan, Z., 2008. New refinements and validation of the MODIS land-surface temperature/emissivity products. Remote Sensing of Environment, 112(1), pp. 59-74.

Wan, Z., and J. Dozier, 1996. A generalized split-window algorithm for retrieving land-surface temperature from space. IEEE Transactions on Geoscience \& Remote Sensing, 34(4), pp. 892-905.
Wan, Z., 1999. MODIS Land Surface Temperature Algorithm Theoretical Basis Documentation.

Wang, K., J. Wang, P. Wang, M. Sparrow, J. Yang, and H. Chen, 2007. Influences of urbanization on surface characteristics as derived from the Moderate-Resolution Imaging Spectroradiometer: A case study for the Beijing metropolitan area. Journal of Geophysical Research: Atmospheres, 112(D22), p. D22S06.

Wang, W. C., Z. Zeng, and T. R. Karl, 1990. Urban heat islands in China. Geophysical Research Letters, 17(13), pp. 2377-2380. Ward, K., S. Lauf, B. Kleinschmit, and W. Endlicher, 2016. Heat waves and urban heat islands in Europe: A review of relevant drivers. The Science of the total environment, 569-570, pp. 527-539.

World Health Organization, 2010. 2010 global progress report on the implementation of the WHO framework convention on Tobacco control. Rep. Mccarthy.

Wu, S., Y. Yin, D. Zheng, and Q. Yang, 2005. Aridity/humidity status of land surface in China during the last three decades. Science in China Series D: Earth Sciences, 48(9).

Yuan, F., and M. E. Bauer, 2007. Comparison of impervious surface area and normalized difference vegetation index as indicators of surface urban heat island effects in Landsat imagery. Remote Sensing of Environment, 106(3), pp. 375-386.

Zhao G, Dong J, Liu J, et al, 2017. Different Patterns in Daytime and Nighttime Thermal Effects of Urbanization in Beijing-Tianjin-Hebei Urban Agglomeration. Remote Sensing, 9(2), p. 121.

Zhou, B., D. Rybski, and J. P. Kropp, 2013. On the statistics of urban heat island intensity. Geophysical Research Letters, 40(20), pp. 5486-5491.

Zhou, D., S. Zhao, S. Liu, L. Zhang, and C. Zhu, 2014. Surface urban heat island in China's 32 major cities: Spatial patterns and drivers. Remote Sensing of Environment, 152, pp. 51-61.

Zhou, D., S. Zhao, L. Zhang, G. Sun, and Y. Liu, 2015. The footprint of urban heat island effect in China. Scientific reports, 5, p. 11160

Zhou, L., and J. E. Hansen, 2004. Evidence for a significant urbanization effect on climate in China. Proceedings of the National Academy of Sciences, 101(26), pp. 9540-9544. 